\title{
Covalently Functionalized Hexagonal Boron Nitride Nanosheets via Nitrene Addition
}

\author{
Toby Sainsbury ${ }^{1}$, Amro Satti ${ }^{2}$, Peter May ${ }^{1}$, Arlene O’Neill ${ }^{1}$, Valeria Nicolosi ${ }^{3}$, Yurii K. Gun'ko ${ }^{2}$, and Jonathan N. Cole- \\ $\operatorname{man}^{1 *}$ \\ ${ }^{1}$ School of Physics and CRANN, Trinity College Dublin, Dublin 2, Ireland \\ ${ }^{2}$ School of Chemistry and CRANN, Trinity College Dublin, Dublin 2, Ireland \\ ${ }^{3}$ Department of Materials, University of Oxford, Parks Road, Oxford OX1 3PH, UK
}

KEYWORDS: Boron Nitride, surface functionalization, exfoliation, nanosheets, nitrene, polymer nanocomposite.

\begin{abstract}
Exfoliated h-BN nanosheets were covalently functionalized via a one-step nitrene addition using azide precursor molecules. Functionalized h-BN nanosheets were found to exhibit markedly enhanced dispersability relative to pristine h-BN in a range of organic solvents. Extension of the functionalization methodology allowed the covalent attachment of polymer chains to the surface of h-BN nanosheets. Polymer nanocomposites were prepared using the molecularly-functionalized h-BN nanosheets within a polycarbonate (PC) matrix, while h-BN nanosheets functionalized with poly(bisphenol A-co-epichlorohydrin) (PBCE) chains were employed within a PBCE matrix. In both cases the mechanical properties were studied. Significant increases in moduli, strength and ductility of the functionalized h-BN nanocomposites indicated enhanced reinforcement of the functionalized h-BN filler materials over pristine h-BN. The implications of tuning the surface chemistry of exfoliated nanosheets exactly to the chemistry of a host polymer matrix are considered.
\end{abstract}

Due to its exciting electrical, thermal and mechanical properties, ${ }^{1}$ the last few years have seen a surge of interest in the exfoliation of graphene..$^{2, \underline{3}}$ More recently, attention has shifted to other layered compounds, notably hexagonal boron nitride (h-BN) and the transition metal dichalcogenides. $\frac{4-6}{}$ Several reports have emerged describing the exfoliation and solubilization of h-BN. ${ }^{5-9}$ However, perhaps due to its perceived inert nature, exfoliated h-BN has not been covalently functionalised. In contrast, a number of groups have described the covalent modification of graphene. $\underline{10} \underline{11}$ This is important as such modification can impart chemical functionality and facilitate integration of the exfoliated material within polymer systems. Due to the high intrinsic strength of $\mathrm{h}-\mathrm{BN}, \underline{12}$ such systems could be the basis of future high performance composites. It is therefore highly attractive to investigate controlled synthetic methodologies for the covalent chemical functionalization of the planar h-BN lattice. The covalent functionalization of h$\mathrm{BN}$ with organic molecules will of course significantly alter the surface chemistry and hence dictate the nature of the interaction between h-BN and liquid or matrix materials. It is therefore of interest to controllably graft organic functional molecules of choice to exfoliated h-BN. Previously, nitrene addition has been used to covalently functionalize graphene and CNTs via the formation of aziridine ring species. ${ }^{0,}, 13$ Even though BN allotropes are known to exhibit excellent chemical stability in strongly acidic and basic conditions,,$\frac{14}{14}$ might be expected that the generation of a highly reactive nitrene species in the presence of exfoliated h-BN could result in covalent functionalization of the h-BN sheet.
In this communication, we report the covalent chemical functionalization of $\mathrm{h}-\mathrm{BN}$ nanosheets using reactive nitrene radicals. Detailed characterization of the functionalized h-BN was performed to verify the covalent nature of the interaction. The functionalization methodology was extended to covalently attach polymer chains to the surface of h-BN nanosheets and use these polymer-functionalized $\mathrm{BN}$ nanosheets as filler within polymer composites.

Hexagonal BN (h-BN) was exfoliated in N-methylpyrrolidone (NMP) as described previously. ${ }^{6}$ The organo-azide precursor, 4-methoxybenzyloxycarbonyl azide, was added and the reactive nitrene intermediate generated by thermolysis. Thermolysis was confirmed by a color change from milkywhite to dark brown as well as the generation of $\mathrm{N}_{2}$ bubbles from the suspension. Nitrene radicals are expected to attack $B$ atoms in the h-BN lattice leading to $\mathrm{B}-\mathrm{N}$ bond cleavage. This will yield methoxyphenyl carbamate (MPC) bound to the hBN lattice via B-N bond formation, as depicted by the scheme in Figure 1a.

Surface functionalization of any nano-material is expected to have a significant effect on its dispersability in organic solvents. $.^{15}, 16$ Indeed, while pristine h-BN exhibits limited solubility in ethanol, $\underline{6}$ methoxyphenyl carbamate-BN (MPC-BN) was readily dispersed in ethanol (1 min sonication, $24 \mathrm{hr}$ sedimentation)(Figure 1b). Measurements of absorbance $(\lambda=300$ $\mathrm{nm}$ ) as a function of dispersed concentration (as estimated by filtration and weighing, see SI) showed linear behavior giving an extinction coefficient of close to $\varepsilon=3000 \mathrm{mLmg}^{-1} \mathrm{~m}^{-1}$ for both pristine and functionalized h-BN (figure $3 c$, inset). UV- 
Vis absorption of the freshly sonicated suspensions indicated distinctly increased extinction for the MPC-BN nanosheets as compared to the pristine h-BN as shown in Figure 1c. Knowledge of $\varepsilon$ allowed the measurements of concentrations of up to $0.5 \mathrm{mgmL}^{-1}$ in ethanol (Figure 1c, see also SI). (a)

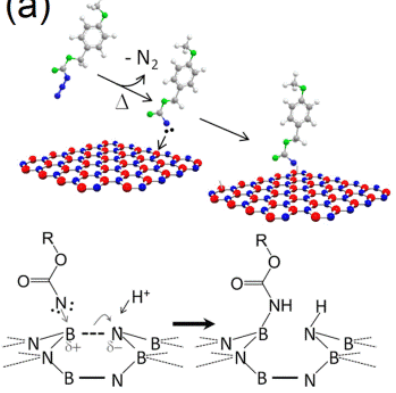

(c)

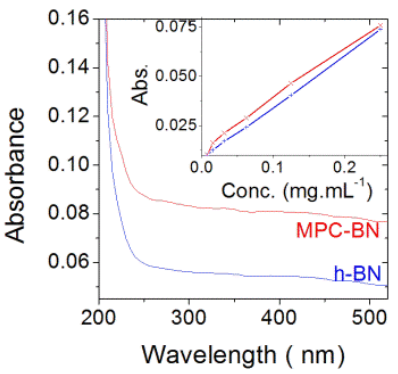

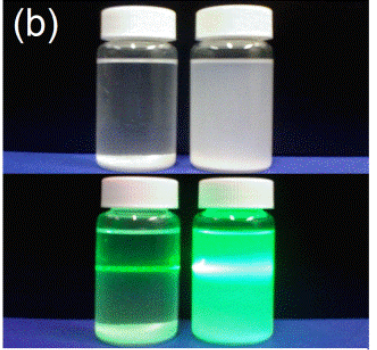
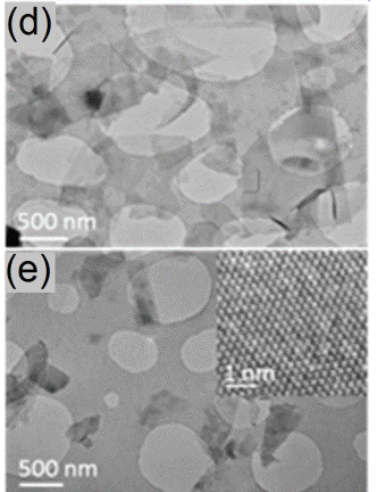

Figure 1. a) Schematic illustration of grafting of nitrene species to h-BN nanosheets. b) Dispersions of h-BN (left) and MPC-BN (right) in ethanol. Bottom: under laser illumination. c) Absorbance spectra for BN and MPC-BN (1mm cuvette). Inset: LambertBeer plot. TEM images of h-BN nanosheets d) before and e) following MPC functionalization. Inset; HRTEM image of MPC-BN hexagonal lattice.

In addition, the MPC-BN can be dispersed in the solvents $\mathrm{CHCl}_{3}$, cyclohexylpyrrolidone and dimethylformamide at concentrations 2-3 times higher than can be achieved for pristine h-BN (see supporting info.). In contrast the functionalized h$\mathrm{BN}$ becomes less dispersible in the solvents isopropanol, tetrahydrafuran and toluene. Such changes are classic indicators of the change in surface chemistry after functionalization.

TEM analysis of NMP dispersed $\mathrm{h}-\mathrm{BN}$, before and after functionalization, showed no observable damage to the flakes. However, after functionalization, the MPC-BN nanosheets appeared marginally more aggregated, existing in slightly thicker more disordered flakes (Figure 1d\&e). The HR-TEM in the inset of Figure 2e shows that the crystal structure of the MPC-BN has not been compromised by the reaction. The fact that large quantities of defects and holes are not observable suggests the degree of functionalization to be low. This is encouraging, as low-level functionalization is desirable in order to retain intrinsic properties of the h-BN sheets.

Infra-red spectroscopy was used to characterize the MPC molecules bound to the surface of h-BN (see Figure 2 and supporting information). Following the nitrene functionalization procedure, bands consistent with the MPC molecule are now evident in the spectrum of the MPC-BN. Methoxy and methylene $\mathrm{C}-\mathrm{H}$ bands are observed at $2962 \mathrm{~cm}^{-1}$ and 2926, $2855 \mathrm{~cm}^{-1}$, while carbamate and methoxy C-O-C bands are present at $1260 \mathrm{~cm}^{-1}, 1092 \mathrm{~cm}^{-1}$ and $1021 \mathrm{~cm}^{-1}$. Significantly, intense azide bands observed at $2188 \mathrm{~cm}^{-1}$ and $2131 \mathrm{~cm}^{-1}$ in the free molecule, are absent in MPC-BN, as is any indication of a free amine group in the $3400 \mathrm{~cm}^{-1}$ region. We would expect to see such groups if the molecules were physisorbed.

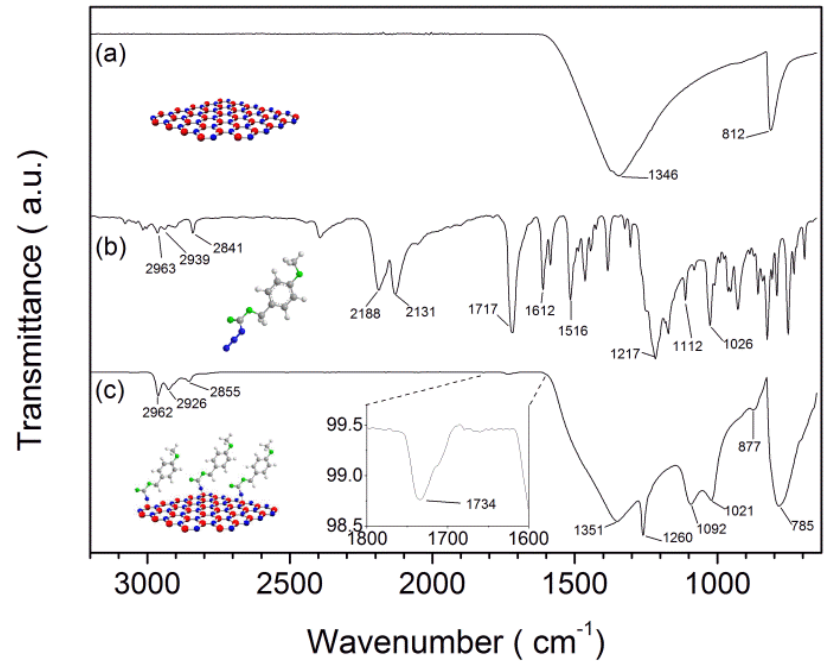

Figure 2. FTIR spectra of a) pristine h-BN, b) methoxybenzyloxycarbonyl azide and c) MPC-BN

The band at $1734 \mathrm{~cm}^{-1}$ is assigned to the carbamate $\mathrm{C}=\mathrm{O}$ adjacent to the $\mathrm{N}$ bound to the h-BN boron-atom. The significant loss in intensity and shift of the $\mathrm{C}=\mathrm{O}$ vibration from $1718 \mathrm{~cm}^{-1}$ in the starting methoxyphenyl azido formate to $1734 \mathrm{~cm}^{-1}$ in the bound molecule is consistent with covalent bonding..,$\underline{11}$ Thus, the presence of the molecular signature of the MPC molecule along with additional bonding features strongly supports the assertion of covalent nitrene addition to the h-BN lattice.

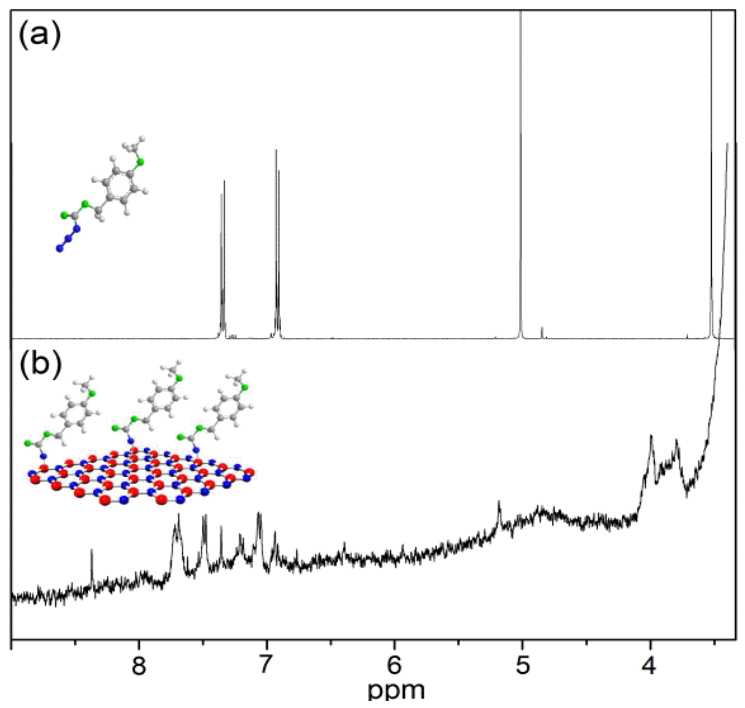

Figure 3. ${ }^{1} \mathrm{H}-\mathrm{NMR}$ spectra of a) methoxyphenylcarbonyl azide molecule b) MPC-BN, both in $\mathrm{CDCl} 3$.

${ }^{1} \mathrm{H}-\mathrm{NMR}$ was also used to characterize the MPCfunctionalized h-BN. The spectrum of the free MPC molecule exhibits peaks assigned to aromatic protons between 6.8 and 7 ppm, while peaks at 3.4 and 5 ppm correspond to the methoxy and methylene protons respectively (Figure $3 \mathrm{a}$ ). In the spectrum of MPC-BN (Figure 3b), multiplets in the aromatic region 7-8 ppm, corresponding to the aromatic protons in the parent molecule (Figure 3a). Peaks at 5.1 and $4.0 \mathrm{ppm}$ are 
assigned to methylene protons adjacent to the carbamate and to the methoxy protons respectively.

The appearance of a weak peak at $8.4 \mathrm{ppm}$ is assigned to the carbamate $\mathrm{N}-\mathrm{H}$ proton adjacent to the B-N bond. The absence of amino protons in the spectrum, expected at $6.0 \mathrm{ppm}$, further corroborates direct nitrene binding and precludes simple physisorption of the free MPC-amine molecule at the surface of hBN nanosheets. This evidence supports our hypothesis that reactive nitrene species generated from the azide thermolysis bind to the h-BN nanosheets via a nitrene addition reaction.

In order to estimate the degree of functionalization, we performed thermogravimetric analysis (TGA) on both h-BN and the MPC-BN (Figure 4). While the mass of h-BN remained roughly constant to $\sim 800^{\circ} \mathrm{C}$, approximately $4 \%$ of MPC-BN mass was lost in the temperature range $250-600^{\circ} \mathrm{C}$. This is consistent with the removal of covalently bound organic molecules at the surface of the h-BN nanosheets. Assuming a molecular weight of $179 \mathrm{~g} / \mathrm{mol}$ for the MPC, this mass loss is equivalent to one functional group per $\sim 200 \mathrm{BN}$ pairs. This means the functional groups are separated by $\sim 4-5 \mathrm{~nm}$ on average. This relatively sparse coverage explains why virtually no damage was observed in the HRTEM images.

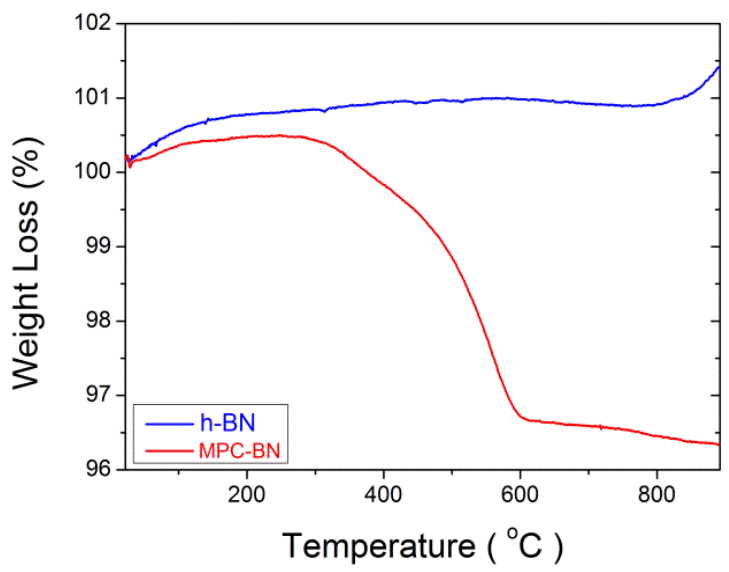

Figure 4. Thermogravimetric traces of a) pristine h-BN and b) MPC-BN.

One area where surface functionalization will be critically important will be to compatibilize the h-BN surface with polymers to facilitate composite formation. Such surface engineering is critical for reinforcement of polymers where stress transfer at the interface is essential. To investigate the influence of the surface functionalization of the h-BN a series of polymer composites were prepared. Initially polycarbonate (PC) was chosen as the host matrix because its chemical similarity to the grafted MPC should result in a reasonable functional group-matrix interaction.

We prepared composite dispersions of both h-BN and MPC$\mathrm{BN}$ (both with mass fraction $\mathrm{M}_{\mathrm{f}}=0.1 \mathrm{wt} \%$ ) mixed with polycarbonate in THF. These were dropcast to form thin films and characterized mechanically with the stress strain curves shown in figure 5a. Addition of $0.1 \% \mathrm{~h}-\mathrm{BN}$ to $\mathrm{PC}$ resulted in small increases in modulus and strength. Interestingly, the ductility increased dramatically from $\sim 15 \%$ to $\sim 70 \%$. However, on addition of $0.1 \mathrm{wt} \%$ MPC-BN, larger increases in modulus (1.51 to $1.86 \mathrm{GPa})$ and strength $(64.4$ to $80 \mathrm{MPa})$ were observed. In addition, a further increase in ductility to $90 \%$ was observed. We note that because the ductility is enhanced for both h-BN and MPC-BN composites, this suggests that residual solvent may act as a plastifying agent in both cases.

However, compatiblization could be made more general, if a small linker molecule could be covalently bonded to the h-BN nanosheet, to which further molecules of choice could also be attached. To this end, we have bound pentanoic acid directly to the h-BN. This was achieved by grafting azidopentanoic acid molecules to the surface of the h-BN via nitrene addition as before (see SI for more detail). Resultant terminal carboxylic acid groups are then amenable to coupling chemistry with alcohol or amine groups and hence the attachment of a range of polymer chains. With composite reinforcement in mind, we focus on the attachment of the polymer, poly(bisphenol A-coepichlorohydrin), to give PBCE-BN as described in the supporting information.
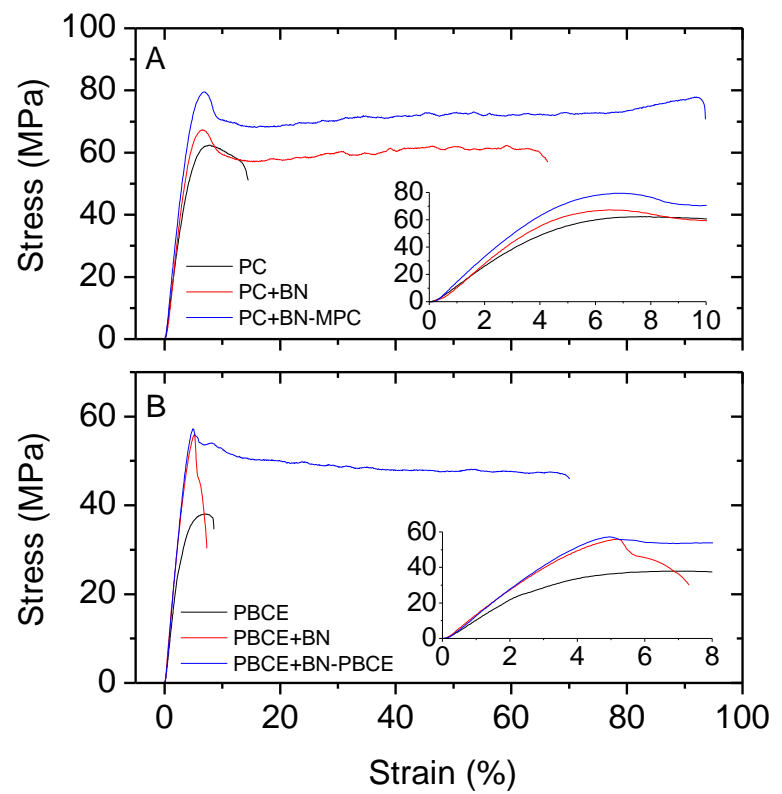

Figure 5. Stress strain curves for $A) P C, P C$ mixed with $B N$ $(0.1 \mathrm{wt} \%)$ and PC mixed with MPC-BN $(0.1 \mathrm{wt} \%)$ and B) PBCE, PBCE mixed with BN $(0.1 \mathrm{wt} \%)$ and PBCE mixed with PBCE-BN $(0.1 \mathrm{wt} \%)$. Insets: the low strain region.

We emphasise the importance of covalently attaching a polymer chain rather than a small molecule from the perspective of mechanical reinforcement. For reinforcement to be maximised, achieving high interfacial stress transfer is critical. The attachment of polymer chains to the surface of BN nanosheets would facilitate entanglement of bound chains within the matrix. This should give good stress transfer and hence significant reinforcement. ${ }^{17}$ In addition, because significant energy would be required to disentangle the grafted chains form the matrix, one might expect the toughness to be increased. However we note that for entanglement to occur, the enthalpy of mixing of the grafted polymer and matrix must be extremely low. ${ }^{18}$ In practise, significant entanglement will only occur if the grafted and matrix polymers are identical. For this reason we chose to study the mechanical properties of $\mathrm{PBCE}-\mathrm{BN}$ in a PBCE matrix.

We prepared composites of both $\mathrm{h}-\mathrm{BN}$ and PBCE-BN in a PBCE matrix with mass fractions of $\mathrm{M}_{\mathrm{f}}=0.1 \%$ and performed tensile testing to assess the mechanical reinforcement. For both h-BN and PBCE-BN, the modulus increased from $\mathrm{Y}=1.2$ GPa for the polymer to $1.5 \mathrm{GPa}$ for the $0.1 \%$ composite. This 
is equivalent to a rate of increase of $\mathrm{dY} / \mathrm{dM}_{\mathrm{f}} \approx 300 \mathrm{GPa}$. This is about $30 \%$ of the theoretical maximum value of $1 \mathrm{TPa}$ (set by the modulus of h-BN) and is roughly as expected for randomly oriented nanosheets in the polymer matrix. ${ }^{19,20}$ Interestingly, the fact that the composites have such similar moduli suggests that stress transfer is not enhanced for the polymergrafted h-BN nanosheets. This does not necessarily mean that the grafted polymer chains do not transfer stress effectively. On the contrary, it may be that stress is transferred well to the nanosheets specifically for the polymer used here. It is known that aromatic conjugated molecules and polymers strongly interact with BNNTs due to the pseudo-delocalized pi system at the surface of BNNTs. ${ }^{21}$. This may result in a higher than expected stress transfer specific to matrices such as PC and PBCE. It may be that functionalization with non-conjugated chains for compatibilization with a non-conjugated matrix will result in better stress transfer relative to the unfunctionalized sheets.

The strength increased from $\sigma \approx 38 \mathrm{MPa}$ for the polymer to 55 and $57 \mathrm{MPa}$ for the $\mathrm{h}-\mathrm{BN}$ and PBCE-BN filled composites. This is equivalent to a rate of increase of $d \sigma / \mathrm{dM}_{\mathrm{f}} \approx 18 \mathrm{GPa}$, comparable to the best graphene-polymer composites. ${ }^{21-23}$ Again the fact that both composites display similar strengths suggests that the interfacial shear strength is not improved by the polymer grafting. However, we note that the ductility for both polymer and h-BN reinforced polymer are similar at 7 $8 \%$. However, the PBCE-BN filled polymer displays a ductility of $\sim 70 \%$, a dramatic increase. Because of this large increase in ductility, the tensile toughness of the BN-PBCE composite was 13 times larger than the other two samples (34 MPa versus $2.5 \mathrm{MPa}$ for the polymer). As described above, this large increase in toughness can be attributed to the energy dissipated in disentangling the grafted polymer chains from the matrix polymer. This demonstrates the importance of the grafting of polymer chains in general and the suitability of h-BN in particular as a reinforcement material. We note that none of these systems are optimised. Future work will involve developing composites with higher loading levels to give significantly increased modulus and strength.

In summary, covalent surface functionalization of $\mathrm{BN}$ nanosheets has been demonstrated via nitrene addition. The grafting of organic molecules significantly enhanced the dispersibility of the nanosheets within organic solvents and facilitated chemical compatibilization of the h-BN nanosheets within polymer matrices. We expect that the functionalization scheme could be modified to allow a polymer of choice to be attached to the BN nanosheets. This would allow the mechanical reinforcement of any polymer matrix.

\section{ASSOCIATED CONTENT}

Supporting Information. Details of Boron Nitride sample preparation and characterization using FTIR, TGA, UV-Vis, and me- chanical testing are included, Figures S1-S10. This material is available free of charge via the Internet at http://pubs.acs.org.

\section{AUTHOR INFORMATION}

\section{Corresponding Author}

*colemaj@tcd.ie

\section{ACKNOWLEDGMENT}

TS, JC would like to thank the European Research Council for funding through the grant SEMANTICS.

\section{REFERENCES}

1. Geim, A. K. Science 2009, 324, 1530.

2. Park, S.; Ruoff, R.S. Nat Nanotechnol 2009, 4, 217.

3. Ruoff, R. Nat Nanotechnol 2008, 3, 10.

4. Rao, C.N.R.; Nag, A. Eur. J. Inorg. Chem. 2010, 4244

5. Zhi, C. Y.; Bando, Y.; Tang, C. C.; Kuwahara, H.; Golberg, D. Adv. Mater. 2009, 21, 2889.

6. Coleman, J. N. et. al. Science 2011, 331, 568.

7. Han, W. Q.; Wu, L. J.; Zhu, Y. M.; Watanabe, K.; Taniguchi, T. Appl. Phys. Lett. 2008, 93.

8. Lin, Y.; Williams, T. V.; Connell, J. W. J. Phys. Chem. Lett. 2010, $1,277$.

9. Warner, J. H.; Rummeli, M. H.; Bachmatiuk, A.; Buchner, B. ACS Nano 2010, 4, 1299.

10. He, H. K.; Gao, C. Chem. Mater. 2010, 22, 5054.

11. Niyogi, S.; Bekyarova, E.; Itkis, M. E.; McWilliams, J. L.; Hamon, M. A.; Haddon, R. C. J. Am. Chem. Soc. 2006, 128, 7720 .

12. Wei, X. L.; Wang, M. S.; Bando, Y.; Golberg, D. Adv. Mater. 2010, 22, 4895 .

13. Holzinger, M.; Abraha, J.; Whelan, P.; Graupner, R.; Ley, L.; Hennrich, F.; Kappes, M.; Hirsch, A. J. Am. Chem. Soc. 2003, $125,8566$.

14. Zhi, C. Y.; Bando, Y.; Terao, T.; Tang, C. C.; Kuwahara, H.; Golberg, D. Chem. Asian J. 2009, 4, 1536.

15. Amiran, J.; Nicolosi, V.; Bergin, S. D.; Khan, U.; Lyons, P. E.; Coleman, J. N. J. Phy. Chem. C 2008, 112, 3519.

16. Sun, Y. P.; Fu, K. F.; Lin, Y.; Huang, W. J. Acc. Chem. Res. 2002, 35, 1096.

17. Blake, R.; Gun'ko, Y. K.; Coleman, J.; Cadek, M.; Fonseca, A.; Nagy, J. B.; Blau, W. J. J. Am. Chem. Soc. 2004, 126, 10226.

18. Rubinstein, M.; Colby, R. H. Polymer Physics; First ed.; Oxford University Press: Oxford, 2003.

19. Padawer, G. E.; Beecher, N. Polym. Eng. and Sci. 1970, 10, 185.

20. Rosenthal, J. Polym. Compos. 1992, 13, 462.

21. Zhi, C.; Bando, Y.; Tang, C.; Honda, S.; Sato, K.; Kuwahara, H.; Golberg, D. J. Phys. Chem. B 2006, 110, 1525.

22. Potts, J. R.; Dreyer, D. R.; Bielawski, C. W.; Ruoff, R. S. Polymer 2011, 52, 5 .

23. Sengupta, R.; Bhattacharya, M.; Bandyopadhyay, S.; Bhowmick, A. K. Prog. Polym. Sci. 2011, 36, 638.

24. Verdejo, R.; Bernal, M. M.; Romasanta, L. J.; Lopez-Manchado, M. A. J. Mater. Chem. 2011, 21, 3301. 


\section{Covalently Functionalized Hexagonal Boron Nitride Nanosheets via Nitrene Addition}

We describe the covalent functionalization of exfoliated hexagonal Boron Nitride (h-BN) nanosheets via nitrene addition. Molecules grafted to the surface of the nanosheets impart significant enhancement of their dispersion in a range of organic solvents and allow the surface chemistry of the nanosheets to be tuned to allow the covalent attachment of polymer chains. Integration of molecularly functionalized h-BN nanosheets within a Polycarbonate matrix is demonstrated and was found to exhibit significant increases in mechanical properties. This integration methodology was further extended by the covalent modification of the h-BN nanosheets with polymer chains of a polycarbonate analogue, poly(Bisphenol-A-coepichlorohydrin)(PBCE) and the integration of the polymer modified h-BN within the PBCE matrix. Identical matching of surface functionalization with a host matrix was found to further enhance the mechanical properties. This functionalization methodology provides a basis for further studies to integrate exfoliated $\mathrm{h}-\mathrm{BN}$ within polymer matrices and demonstrates the potential of this one dimensional nanomaterial.

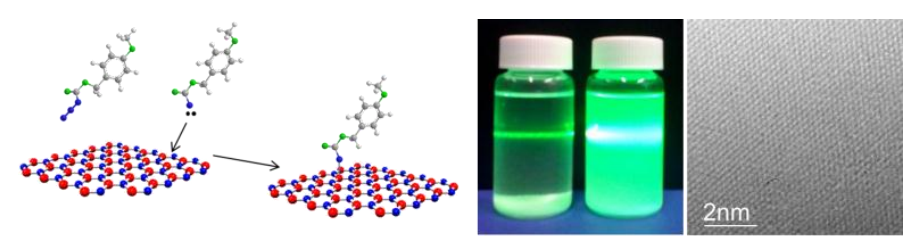

